\title{
Enforcing Sets of Formulas in Structured Argumentation
}

\author{
AnneMarie Borg ${ }^{1}$, Floris Bex ${ }^{1,2}$ \\ ${ }^{1}$ Department of Information and Computing Sciences, Utrecht University \\ ${ }^{2}$ Tilburg Institute for Law, Technology, and Society, Tilburg University \\ \{A.Borg, F.J.Bex\}@UU.nl
}

\begin{abstract}
Enforcement, adjusting an argumentation framework such that a certain set of arguments becomes acceptable, is an important research topic within the study of dynamic argumentation, but one that has been little studied for structured argumentation. In this paper we study enforcement in a general structured argumentation setting. In particular, we study conditions on the argumentation setting and the knowledge base that ensure (or prevent) the acceptability of sets of formulas for structured argumentation frameworks.
\end{abstract}

\section{Introduction}

Argumentation is a dynamic process, in which derived conclusions have to be revised in view of new information. However, basic abstract Argumentation Frameworks (AFs) - arguments and the attacks between them (Dung 1995) - and their conclusions - set(s) of arguments (extensions) that can collectively be accepted - are static. Dynamic approaches to abstract argumentation have been studied, such as enforcement (Baumann and Brewka 2010): given a set of arguments that we want to accept, how to change the argumentation framework in such a way that this set becomes (part of) an extension in the resulting framework?

Although enforcement has been extensively studied for abstract argumentation (see (Doutre and Mailly 2018) for an overview), not much research has been conducted on enforcement for structured argumentation, where arguments can be constructed by inferring conclusions (formulas) from a knowledge base (a set of formulas). In abstract argumentation, dynamics is often modeled by adding arguments/attacks, but in structured argumentation it is not possible to simply add specific arguments/attacks without directly influencing other arguments/attacks in the framework. The structure of individual arguments means that there is a subargument relation, for example, when an argument is based on a subset of the premises (formulas) of another argument. Thus, adding an argument means that its sub-arguments have to be added as well. Similarly, because attacks are dependent on the formulas in the arguments, adding an attack could mean that we have to add an argument as well. Hence, in a structured setting we should define enforcement in terms of formulas instead of in terms of arguments: given a set of formulas that you want to accept, how to change the framework in such a way that this set becomes (part of) the conclusions of an extension in the resulting framework?

For our study of enforcement of sets of formulas, we take a general approach to structured argumentation (Borg and Straßer 2018) so the results hold for many of the well-known approaches (Besnard et al. 2014). We allow formulas to be added to the knowledge base, and show under which conditions on the attack relation and on the new information in the knowledge base a set of formulas can be enforced. We see that a set of formulas can always be enforced if it can be derived from a consistent set of formulas and either (i) that set is maximally consistent; or (ii) the acceptance relation is monotonic; or (iii) the acceptance relation satisfies noninterference and the set does not share any information with the already existing information. To the best of our knowledge, this is the first study on enforcement of sets of formulas in a general structured argumentation setting.

Our study of enforcement in a structured argumentation setting is not only interesting from a theoretical perspective. There are many real-world applications of structured argumentation, including those in legal reasoning (Prakken 2020), crime and forensics (Bex, Testerink, and Peters 2016; Odekerken and Bex 2020), medicine (Čyras et al. 2020; Zeng et al. 2020), technologies for behaviour change (Chalaguine et al. 2018) and debunking fake news (Visser, Lawrence, and Reed 2020). In these application domains, dynamics and specifically enforcement for structured argumentation is imperative. For example, when reasoning in legal or medical cases we want to know whether new observations can enforce a new (legal/medical) conclusion or whether we can stop looking for more evidence. Similarly, in persuasive dialogues for behaviour change or about fake news, it makes sense to enforce one's conclusion in as short a dialogue as possible, since in the case of long dialogues the user will simply disengage.

We start our paper by further illustrating the need for enforcement in a real-life application of structured argumentation (Section 2). The setting from (Borg and Straßer 2018) and enforcement for abstract argumentation (Baumann and Brewka 2010) are recalled in Section 3. Then, in Section 4, enforcement in a structured setting are introduced. Finally, we discuss the implications of some of the assumptions and restrictions made in the paper in Section 5 and related work in Section 6. We conclude in Section 7. 


\section{Enforcement in Real-world Applications}

At the Dutch National Police several argumentation-based applications are being deployed (Bex, Testerink, and Peters 2016). These applications are aimed at assisting the police at working through high volume tasks, leaving more time for tasks that require human attention. For example, there is an application that helps Dutch citizens to file a complaint on online trade fraud and can then determine whether it is a case of fraud (Odekerken, Borg, and Bex 2020), and an application helps the police to identify malafide webshops (Odekerken and Bex 2020). Each of these applications is based on an inquiry process in which the system gathers information until the argumentation component (which is based on a variation of $\mathrm{ASPIC}^{+}$(Prakken 2010)) determines that the derived conclusion will not change if more information is added (i.e., the conclusion is stable).

Take, for example, the application for online trade fraud. This system tries to determine whether, given the information that the citizen has provided, the conclusion for fraud can be stably accepted (i.e., it is currently accepted and will remain so with any future expansion of the knowledge base), or not (e.g., no (accepted) argument for fraud is possible given the current knowledge base, or the acceptance of fraud might still change when new information is obtained). Essentially, the question is whether the conclusion fraud can be enforced for all possible expansions of the knowledge base, and if so, what information is necessary for this enforcement. The application contains a rule-base (based on Dutch fraud law) and a set of queryables, formulas that can be added to the knowledge base and from which (given the rulebase) it might be possible to build arguments for the conclusion fraud. These queryables are observations that can be gathered by, for example, asking the citizen more questions (e.g., 'how much did you pay to the suspected fraudster?') or querying a database (e.g., 'is the suspected fraudster known to the police?'). The question of enforcement becomes then: which of these queryables should be added to the knowledge base to enforce the conclusion fraud? Since the application (and the other applications mentioned here and in Section 1) are based on structured argumentation frameworks (in the case of the trade fraud application a variation of $\mathrm{ASPIC}^{+}$), the existing literature on enforcement does not help to answer this question. In this paper we therefore study enforcement for structured argumentation in a general setting, the results of which are general enough to be implemented in the police applications as well.

\section{Preliminaries}

In this paper it is assumed that there is a formal language $\mathcal{L}$. Sets of formulas are denoted by $\mathrm{S}, \mathrm{T}$, finite sets of formulas are denoted by $\Gamma, \Delta, \Theta$, formulas are denoted by $\gamma, \delta, \phi, \psi$ and atoms are denoted by $p, q, r$, all of which can be primed or indexed if needed. Furthermore, it is assumed that there is a deducability relation $\vdash \subseteq \wp_{\text {fin }}(\mathcal{L}) \times \mathcal{L}\left(\right.$ where $\wp_{\text {fin }}$ denotes the set of finite subsets).

For the results in this paper it is not necessary to assume all the Tarskian conditions on $\vdash$ (i.e., reflexivity, transitivity and monotonicity). Given a language $\mathcal{L}$, a set of $\mathcal{L}$-formulas
$\mathrm{S}$ and a deducability relation $\vdash$, we denote the closure of $\mathrm{S}$ w.r.t. $\vdash$ by $\mathrm{CN}_{\vdash}(\mathrm{S})=\{\phi \mid \exists \Gamma \subseteq$ S s.t. $\Gamma \vdash \phi\}$. When $\vdash$ is clear from the context it will be omitted.

\subsection{General Argumentation Setting}

Abstract argumentation frameworks (Dung 1995) are pairs $A F=\langle\operatorname{Args}, A\rangle$, where Args is a set of arguments and $A \subseteq$ Args $\times$ Args is an attack relation on those arguments in such frameworks, the arguments are abstract entities with no internal content or structure. In structured argumentation the arguments in an argumentation framework are constructed from a knowledge base and a set of rules and the attack relation is based on the structure of these arguments.

Rather than choosing one of the well-known approaches to structured argumentation (see e.g., (Besnard et al. 2014)) as the framework for this paper, the general argumentation setting from (Borg and Straßer 2018) is taken as the argumentation approach. This allows us to keep the results of the current paper as general as possible and does not require to introduce all the specifics of one particular approach. Moreover, the results apply to some (e.g., ASPIC ${ }^{+}$, $\mathrm{ABA}$ and logic-based argumentation) of the approaches discussed in (Besnard et al. 2014), since it was shown in (Borg and Straßer 2018) that these can be translated into the general setting. We recall here the most important definitions from (Borg and Straßer 2018).

Definition 1 (Argument). Given a set of $\mathcal{L}$-formulas $S$, the set of S-based arguments is denoted by $\operatorname{Args}_{\vdash}(S)$, such that $(\Gamma, \gamma) \in \operatorname{Args}_{\vdash}(S)$ iff $\Gamma \vdash \gamma$ for some $\Gamma \subseteq S$. Given an argument $a=(\Gamma, \gamma) \in \operatorname{Args}_{\vdash}(S), \operatorname{Conc}(a)=\gamma$ denotes the conclusion of $a$ and $\operatorname{Supp}(a)=\Gamma$ denotes the support set of a. Where $\mathrm{S}$ is a set of arguments, $\operatorname{Supps}(\mathrm{S})=\bigcup\{\operatorname{Supp}(a) \mid$ $a \in \mathrm{S}\}$ and $\operatorname{Concs}(\mathrm{S})=\{\operatorname{Conc}(a) \mid a \in \mathrm{S}\}$.

To accommodate argumentative attacks two types of functions are introduced: a contrariness function ${ }^{-}: \mathcal{L} \rightarrow \wp(\mathcal{L})$ that associates each formula with a set of conflicting formulas and a target function $\widehat{\imath}: \wp_{\text {fin }}(\mathcal{L}) \backslash \emptyset \rightarrow \wp_{\text {fin }}(\mathcal{L})$ that associates the support set of each argument with the sets of formulas in which the argument can be attacked.

Example 1. Let $\mathcal{L}$ be a formal language and $\vdash_{\mathrm{CL}}$ the consequence relation from classical logic (CL). Some examples of the contrariness and target function are:

negation (ᄀ): $\bar{\phi}=\{\neg \phi\} \quad$ conflict: $\bar{\phi}=\{\psi \mid \psi, \phi \vdash \perp\}$ identity (id): $\widehat{\Gamma}=\Gamma \quad$ conjunction: $\widehat{\Gamma}=\left\{\bigwedge \Gamma^{\prime} \mid \Gamma^{\prime} \subseteq \Gamma\right\}$.

An argumentation setting is based on the deducability relation, the contrariness function and the target function.

Definition 2 (Setting). An (argumentation) setting is a triple $A S_{\vdash}=(\vdash,-, \cdot)$. A setting based on $S \subseteq \mathcal{L}$ is given by $A S_{\vdash}(S)=(S, \vdash, \cdot, \cdot)$.

Attacks between arguments in a setting are based on the contrariness function and the target function:

Definition 3 (Attacks). Let $A S_{\vdash}(S)$ be a setting and let $a=$ $(\Gamma, \gamma) \in \operatorname{Args}_{\vdash}(S)$ and $b=(\Delta, \delta) \in \operatorname{Args}_{\vdash}(S)$. Then $a$ attacks $b$ (in $\phi)$ iff there is $a \phi \in \widehat{\Delta}$ such that $\gamma \in \bar{\phi}$.

Given a setting the corresponding argumentation framework can be defined. 


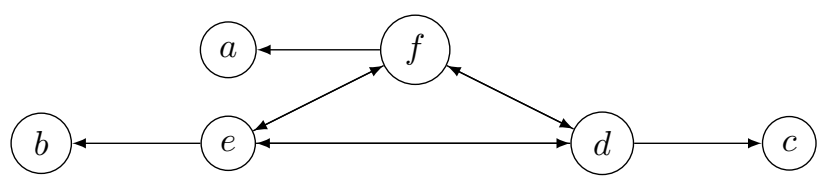

Figure 1: Graphical representation of part of the argumentation framework from Example 2.

Definition 4 (Argumentation framework). Given a setting $A S_{\vdash}(S)$, the corresponding argumentation framework is the pair $A F\left(A S_{\vdash}(S)\right)=\left\langle\operatorname{Args}_{\vdash}(S), A\right\rangle$, where $\operatorname{Args}_{\vdash}(S)$ is the set of $S$-based arguments and $(a, b) \in A$ iff $a, b, \in \operatorname{Args}_{\vdash}(S)$ and $a$ attacks $b$.

Example 2. Let $A_{\mathrm{CL}}(S)=\left(S, \vdash_{\mathrm{CL}}, \neg\right.$, id $)$ be an argumentation setting, based on classical logic, negation $(\neg)$ as the contrariness function and id as the target function, for $S=\{p, q, \neg p \vee \neg q\}$. Then some of the arguments are:

$\begin{array}{ll}a=(\{p\}, p) & d=(\{p, q\}, \neg(\neg p \vee \neg q)) \\ b=(\{q\}, q) & e=(\{p, \neg p \vee \neg q\}, \neg q) \\ c=(\{\neg p \vee \neg q\}, \neg p \vee \neg q) & f=(\{q, \neg p \vee \neg q\}, \neg p) \\ \text { Here, for example, f attacks } a, d \text { and } e \text { since } p & \in\end{array}$ $\widehat{\operatorname{Supp}(a)} \cap \widehat{\operatorname{Supp}(d)} \cap \widehat{\operatorname{Supp}(e)}$ and $\neg p \in \bar{p}$. Also, d attacks

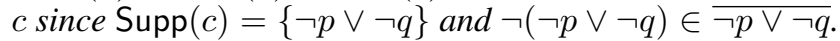
See Figure 1 for a graphical representation of part of the induced argumentation framework.

To determine which arguments can collectively be considered as accepted, Dung-style argumentation semantics (Dung 1995) can be applied to an argumentation framework.

Definition 5 (Argumentation semantics). Let $A F\left(A S_{\vdash}(S)\right.$ ) be an argumentation framework for a setting $A S_{\vdash}(S)$ and let $\mathrm{S} \subseteq \operatorname{Arg}_{\vdash}(S)$ be a set of arguments. Then:

- $\mathrm{S}$ is conflict-free iff there are no $a, b \in \mathrm{S}$ such that a attacks $b$;

- S defends $a \in \operatorname{Args}_{\vdash}(S)$ iff for each attacker $b \in$ $\operatorname{Args}_{\vdash}(S)$ of a there is a $c \in \mathrm{S}$ that attacks $b$;

- $\mathrm{S}$ is admissible (Adm) iff it is conflict-free and it defends every $a \in \mathrm{S}$; and

- $\mathrm{S}$ is complete (Cmp) iff it is admissible and it contains every $a \in \operatorname{Args}_{\vdash}(S)$ it defends.

Some specific complete extensions are:

- $\mathrm{S}$ is preferred (Prf) iff it is $\subseteq$-maximal complete;

- $\mathrm{S}$ is grounded (Grd) iff it is $\subseteq$-minimal complete;

- $\mathrm{S}$ is stable (Stb) iff it is admissible and for all a $\in$ $\operatorname{Args}_{\vdash}(S) \backslash \mathrm{S}$ there is a $b \in \mathrm{S}$ that attacks $a$.

Extensions will be denoted by $\mathcal{E}$, the set of all Semextensions will be denoted by $\operatorname{Sem}\left(A F\left(A S_{\vdash}(S)\right)\right)$ for $\operatorname{Sem} \in$ \{Adm, Cmp, Prf, Grd, Stb\}. ${ }^{1}$

In this paper two types of acceptance relations are considered. These relations are functions with which sets of accepted formulas can be derived from an argumentation framework, given a semantics.

\footnotetext{
${ }^{1}$ In what follows we will sometimes identify $\operatorname{Grd}\left(\AA F\left(A S_{\vdash}(S)\right)\right)$ with its single element.
}

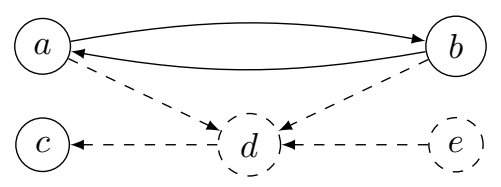

Figure 2: Graphical representation of the argumentation framework from Example 4.

Definition 6 (Acceptance relations). Let $A F\left(A S_{\vdash}(S)\right)$ be an argumentation framework and let Sem $\in\{\mathrm{Grd}, \mathrm{Cmp}, \mathrm{Prf}$, $\mathrm{Stb}\}$. A non-empty set $\mathrm{T}$ of $\mathcal{L}$-formulas is:

- skeptically accepted iff for all $\mathcal{E} \in \operatorname{Sem}\left(A F\left(A S_{\vdash}(S)\right)\right)$, $T \subseteq \operatorname{Concs}(\mathcal{E})$;

- credulously accepted iff there is some $\mathcal{E} \in$ $\operatorname{Sem}\left(A F\left(A S_{\vdash}(S)\right)\right), T \subseteq \operatorname{Concs}(\mathcal{E})$.

Example 3. In the argumentation framework for $A S_{\mathrm{CL}}(S)$, with $S=\{p, q, \neg p \vee \neg q\}$ from Example 2, it can be shown that $\operatorname{Args}(\{p, q\}), \operatorname{Args}(\{p, \neg p \vee \neg q\})$ and $\operatorname{Args}(\{q, \neg p \vee$ $\neg q\})$ are the extensions for Sem $\in\{$ Prf, Stb $\}$.

It follows that a formula $\phi$ is only skeptically accepted for Sem $\in\{\mathrm{Grd}, \mathrm{Cmp}\}$ if it is a tautology in classical logic, $p \vee q$ is skeptically accepted for Sem $\in\{$ Prf, Stb $\}$, since at least $p$ or $q$ is part of each extension and from either $p \vee q$ can be derived in classical logic and all formulas $\psi \in\{p, q, \neg p \vee$ $\neg q\}$ are credulously accepted for Sem $\in\{$ Prf, Stb $\}$.

\subsection{Enforcement for Abstract Argumentation}

In this section, we recall three types of expansions (adding arguments/attacks) and enforcement as introduced in (Baumann and Brewka 2010).

Definition 7. (Expansion, (Baumann and Brewka 2010, Definition 5)) An argumentation framework $A F^{\star}$ is an expansion of the framework $A F=\langle\operatorname{Args}, A\rangle$ iff $A F^{\star}=$ $\left\langle\operatorname{Args} \cup \operatorname{Args}^{\star}, A \cup A^{\star}\right\rangle$, such that Args ${ }^{\star} \neq \emptyset$ and Args $\cap$ $A r g s^{\star}=\emptyset$. Such an expansion is:

- normal iff for all $a$ and $b$, if $(a, b) \in A^{\star}$ then $a \in \operatorname{Args}^{\star}$ or $b \in \operatorname{Args}^{\star}$;

- strong iff it is normal and for all $a$ and $b$, if $(a, b) \in A^{\star}$ then it is not the case that $a \in$ Args and $b \in$ Args $^{\star}$;

- weak iff it is normal and for all $a$ and $b$ if $(a, b) \in A^{\star}$ then it is not the case that $a \in$ Args $^{\star}$ and $b \in$ Args.

Definition 8. (Enforcement, (Baumann and Brewka 2010, Definition 6)) Let $A F=\langle\operatorname{Args}, A\rangle$ be an argumentation framework, Sem a semantics and let $\mathrm{S}$ be a set of arguments, such that $\mathrm{S} \notin \operatorname{Sem}(A F)$. An enforcement of $\mathrm{S}$ is a framework $A F^{\star}$ such that (1) $A F^{\star}$ is $A F$ or a normal expansion of it and (2) $\mathrm{S} \in \operatorname{Sem}\left(A F^{\star}\right)$.

Example 4. Consider the argumentation framework $A F=$ $\langle$ Args, $A\rangle$, where Args $=\{a, b, c\}$ and $A=\{(a, b),(b, a)\}$ shown as the solid part in Figure 2. Now suppose that $A F$ is expanded: $A F^{\prime}=\left\langle\right.$ Args $\left.{ }^{\prime}, A^{\prime}\right\rangle$, where Args ${ }^{\prime}=$ Args $\cup\{d, e\}$ and $A^{\prime}=A \cup\{(a, d),(b, d),(d, c),(e, d)\}$. The resulting graphical representation is the full graph of Figure $2 . A F^{\prime}$ is a normal expansion of AF, since no new attacks are added 
between the arguments of Args. It would be a strong expansion if $(a, d)$ and $(b, d)$ would not be part of $A^{\prime}$ and it would be a weak expansion if $(d, c)$ would not be part of $A^{\prime}$.

\section{Enforcement for Structured Argumentation}

Enforcement in structured argumentation differs from enforcement for abstract argumentation. While in abstract argumentation enforcement is often based on the addition of arguments or attacks, in structured argumentation it is usually not possible to add one specific argument without adding others as well, viz.

Example 5 (Example 2 continued). Consider again the argumentation setting $A S_{\mathrm{CL}}(S)$, with $S=\{p, q, \neg p \vee$ $\neg q\}$. If one would want to add the argument $(\{r\}, r)$ to $A F\left(A S_{\mathrm{CL}}(S)\right), r$ has to be added to $S: S^{\prime}=S \cup\{r\}$. However, then arguments such as $(\{p, r\}, p \wedge r)$ and $(\{r\}, p \vee r)$ are added to $\operatorname{Args}_{\vdash}\left(S^{\prime}\right)$ as well.

Therefore, the notion of an expansion has to be redefined for a structured setting: rather than changing the argumentation framework directly, the argumentation setting on which the framework is based is expanded.

Definition 9 (Expansion, structured argumentation). Let $A F\left(A S_{\vdash}(S)\right)$ be an argumentation framework, for an argumentation setting $A S_{\vdash}(S)$ and some set of formulas $S \subseteq \mathcal{L}$. An expansion for $A F\left(A S_{\vdash}(S)\right)$ is a set of formulas $S^{\prime} \subseteq \mathcal{L}$, such that $S^{\prime} \neq \emptyset$ and $S^{\prime} \cap S=\emptyset$, and the resulting framework is $A F\left(A S_{\vdash}\left(S \cup S^{\prime}\right)\right)=\left\langle\operatorname{Arg}_{\vdash}\left(S \cup S^{\prime}\right), A^{\prime}\right\rangle$, where $(a, b) \in A^{\prime}$ iff $a, b \in \operatorname{Args}_{\vdash}\left(S \cup S^{\prime}\right)$ and a attacks $b$ as defined in Definition 3.

Now enforcement is not about (sets of) arguments, but about (sets of) formulas. Before defining enforcement in terms of sets of formulas, first some useful notions.

Definition 10 ((Strong) (in)consistency). Given an argumentation setting $A S_{\vdash}=\langle\vdash,-, \cdot\rangle$ a set of formulas $S$ and a set of formulas $\Theta \subseteq S$. Then:

- $\Theta$ is A. $S_{-}$-inconsistent iff there is a $\gamma \in \Theta$ for which $\Theta \backslash\{\gamma\} \vdash \gamma^{\prime}$ where $\gamma^{\prime} \in \bar{\gamma}$. $\Theta$ is strongly A $\mathrm{S}_{\vdash}(\mathrm{S})$ inconsistent if $\Theta \subseteq \Theta^{\prime} \subseteq S$ implies that $\Theta^{\prime}$ is inconsistent.

- $\Theta$ is $\mathrm{A} \mathrm{S}_{\vdash}$-consistent iff it is not $\mathrm{A} S_{\vdash}$-inconsistent and $\Theta$ is strongly A.S--consistent if $\Theta^{\prime} \subseteq \Theta$ implies that $\Theta^{\prime}$ is AS $S_{\vdash}$-consistent.

- $\Theta$ is maximal AS $S_{\vdash}$-consistent iff there is no $A S_{\vdash}$ consistent $\Theta^{\prime} \subseteq$ S such that $\Theta \subset \Theta^{\prime}$ and $\Theta$ is maximal strongly $\mathrm{AS}_{\vdash}(\mathrm{S})$-consistent if $\Theta \subsetneq \Theta^{\prime} \subseteq S$ implies that $\Theta^{\prime}$ is not strongly $A S_{\vdash}(S)$-consistent.

We denote $\mathrm{CS}\left(\mathrm{A}_{\vdash} S_{\vdash}(S)\right)$ [MCS $\left.\left(A S_{\vdash}(S)\right)\right]$ for all [maximal] strongly $A S_{\vdash}(S)$-consistent sets of $S$. If the argumentation setting is clear from the context we will write $\mathrm{CS}(S)$ [resp. $\operatorname{MCS}(S)]$.

Remark 1. Since $\vdash$ is not assumed to be monotonic, we use the definition of strong inconsistency from (Brewka, Thimm, and Ulbricht 2019). Note that, when $\vdash$ is monotonic, this definition coincides with the definition of (in)consistency from (Borg and Straßer 2018).
Example 6 (Example 3 continued). In the argumentation framework from the previous subsection, where $A S_{\mathrm{CL}}=$ $\left\langle\vdash_{\mathrm{CL}}, \neg\right.$, id $\rangle$ and $S=\{p, q, \neg p \vee \neg q\}$, the set $S$ is $A S_{\mathrm{CL}-}$ inconsistent. However, every $S^{\prime} \subset S$ is $A S_{\mathrm{CL}}$-consistent. In particular, there are three maximally consistent subsets: $\operatorname{MCS}(S)=\{\{p, q\},\{p, \neg p \vee \neg q\},\{q, \neg p \vee \neg q\}\}$.

In what follows we will assume that the set of formulas to be enforced is strongly $\mathrm{AS}_{\vdash}(\mathrm{S})$-consistent, since otherwise the consistency postulate from (Caminada and Amgoud 2007) could be violated.

Next, we define enforcement for structured argumentation. Enforcing a set of formulas can be done strictly, where the conclusions are exactly that set of formulas, or nonstrictly, where the set of formulas has to be part of the conclusions (cf. (Baumann and Brewka 2010)).

Definition 11 (Enforcement, structured argumentation). Let $A F\left(A S_{\vdash}(S)\right)$ be an argumentation framework and let $T \subseteq \mathcal{L}$ be the set of formulas to be enforced. Then:

- $T$ is [strictly] credulously enforced by the expansion $S^{\prime} \subseteq$ $\mathcal{L}$ if there is some extension $\mathcal{E} \in \operatorname{Sem}\left(A F\left(A S_{\vdash}\left(S \cup S^{\prime}\right)\right)\right.$ such that $[\operatorname{Concs}(\mathcal{E})=\mathrm{CN}(T)] \operatorname{Concs}(\mathcal{E}) \supseteq T$.

- $T$ is [strictly] skeptically enforced by the expansion $S^{\prime} \subseteq$ $\mathcal{L}$ if for all extensions $\mathcal{E} \in \operatorname{Sem}\left(A F\left(A S_{\vdash}\left(S \cup S^{\prime}\right)\right)\right.$ it holds that $[\operatorname{Concs}(\mathcal{E})=\mathrm{CN}(T)] \operatorname{Concs}(\mathcal{E}) \supseteq T$.

Enforcement of a single formula $\phi$ means that $\mathrm{T}=\{\phi\}$ in the definition above. The definition is kept general in that $S^{\prime} \subseteq \mathcal{L}$. Depending on the application $S^{\prime}$ might be restricted. For example, in the online trade fraud example from Section $2 S^{\prime}$ would be restricted to the set of queryables, since these are the only formulas that can be added to the knowledge base.

Example 7 (Example 2 continued). Consider again the framework $A F\left(A S_{\mathrm{CL}}(S)\right)$ for $S=\{p, q, \neg p \vee \neg q\}$. Suppose that $S^{\prime}=S \cup\{r\}$ as in Example 5. For this expanded set it holds that $\operatorname{Prf}\left(A F\left(A S_{\vdash}\left(S^{\prime}\right)\right)\right)=\left\{\operatorname{Args}_{\mathrm{CL}}(\{p, q, r\})\right.$, $\left.\operatorname{Args}_{\mathrm{CL}}(\{p, \neg p \vee \neg q, r\}), \operatorname{Args}_{\mathrm{CL}}(\{q, \neg p \vee \neg q, r\})\right\}$. Therefore $r$ is both credulously and skeptically enforced by $\{r\}$.

In the next sections formal results on whether enforcing a set of formulas is possible are shown. The question of how such a set of formulas should be enforced is left for future work. We start by introducing some useful properties.

\subsection{Relating Sets of Formulas and Sets of Arguments}

Since enforcement is about extensions (sets of arguments) and in the structured setting defined for sets of formulas, sets of formulas and sets of arguments have to be related. In this section results for this relation for static argumentation are presented. ${ }^{2}$

Most of the results in the remainder of the paper will be shown for argumentation settings with the identity (id) target

\footnotetext{
${ }^{2}$ Due to space restrictions we cannot provide proofs in this section. For full proofs we refer to the online technical appendix at: https://nationaal-politielab.sites.uu.nl/ enforcement-structured-argumentation/.
} 
function. ${ }^{3}$ In such settings, when the set of formulas is A.S-consistent, there is only one extension and all conclusions of that extension are skeptically accepted.

Lemma 1. Let $A S_{\vdash}=\langle\vdash, \bar{\cdot}$, id $\rangle$ be an argumentation setting and let $S$ be a strongly $A S_{\vdash}(S)$-consistent set of $\mathcal{L}$ formulas. Then: $\operatorname{Sem}\left(A F\left(A S_{\vdash}(S)\right)\right)=\left\{A \operatorname{Args}_{\vdash}(S)\right\}$ for Sem $\in\{\mathrm{Grd}, \mathrm{Cmp}, \operatorname{Prf}, \mathrm{Stb}\}$, and $\mathrm{CN}(S)$ is skeptically accepted in $A F\left(A S_{\vdash}(S)\right)$.

The next proposition shows how extensions of argumentation frameworks and sets of formulas are related. While the relation between formal argumentation and reasoning with consistent subsets is well-known (Arieli, Borg, and Heyninck 2019), our result here (i.e., Proposition 1) is more general than the existing results. This is the case since we employ the general setting from (Borg and Straßer 2018), but we restrict the setting less than is done in that paper (e.g., it is not assumed that the support set of an argument is consistent, nor that $\vdash$ is transitive). Moreover, we apply the more general notion of inconsistency from (Brewka, Thimm, and Ulbricht 2019), which is also applicable to nonmonotonic $\vdash$. It is, however, assumed that the setting is contrapositable (see also Footnote 3):

Definition 12. (Contrapositable settings (Borg and Straßer 2018, Definition 18)) A setting $\left(\vdash,,^{-}\right)$is contrapositable iff for all $\Theta \in \wp_{\text {fin }}(\mathcal{L})$, if $\Theta \vdash \gamma^{\prime}$ where $\gamma^{\prime} \in \bar{\gamma}$ then for all $\delta \in \Theta$, $(\Theta \cup\{\gamma\}) \backslash\{\delta\} \vdash \delta^{\prime}$ for some $\delta^{\prime} \in \bar{\delta}$. By extension we call $A S_{\vdash}=\langle\vdash, \cdot, \cdot\rangle$ contrapositable if $(\vdash, \bar{*})$ is contrapositable.

Proposition 1. Let $A F\left(A S_{\vdash}(S)\right)$ be such that $A S_{\vdash}(S)=$ $\left\langle\vdash,{ }^{-}, \mathrm{id}\right\rangle$ is contrapositable and let Sem $\in\{\mathrm{Cmp}, \operatorname{Prf}, \mathrm{Stb}\}$ :

1. if $T \in \operatorname{CS}(S)$, then there is some $T^{\prime} \supseteq T$ such that $\operatorname{Args}_{\vdash}\left(T^{\prime}\right) \in \operatorname{Sem}\left(A F\left(A S_{\vdash}(S)\right)\right)$;

2. if $T \in \operatorname{MCS}(S)$, then $\operatorname{Args}_{\vdash}(T) \in \operatorname{Sem}\left(A F\left(A S_{\vdash}(S)\right)\right)$;

3. if $\mathcal{E} \in \operatorname{Sem}\left(A F\left(A S_{\vdash}(S)\right)\right)$ then $\operatorname{Supps}(\mathcal{E}) \in \operatorname{CS}(S)$;

4. if $\mathcal{E} \in \operatorname{Prf}\left(A F\left(A S_{\vdash}(S)\right)\right)$ then $\operatorname{Supps}(\mathcal{E}) \in \operatorname{MCS}(S)$.

The first item states that for any consistent subset there is a superset from which an extension can be constructed and the second item shows that arguments constructed from a maximally consistent subset form an extension. The third item shows that the union of the support set of all arguments in an extension is always consistent and the fourth shows that the support set of the arguments of a preferred extension is a maximally consistent subset. Since enforcement in structured argumentation is defined for sets of formulas, these results show how sets of formulas that should be enforced and extensions can be related.

\subsection{Enforcing Formulas}

Throughout this section we will consider several conditions that make enforcement of sets of formulas (im)possible. In addition to general conditions on, for example, the set of formulas that should be enforced and the acceptance relation, we will discuss the expansion types and the notion of monotonicity from (Baumann and Brewka 2010).

\footnotetext{
${ }^{3}$ See Section 5 for a discussion on this assumption.
}

Skeptical enforcement. The type of acceptance (i.e., skeptical or credulous) determines, partially, the possibility of enforcing a set of formulas. Intuitively, it is easier to enforce a set of formulas credulously, since then only one extension has to be considered, while for skeptical enforcement all extensions have to be considered. Indeed, when the set of formulas that should be enforced can only be derived from sets of formulas that are conflicting with the current knowledge base, the set cannot be skeptically enforced. Before we show this, we introduce the next lemma: ${ }^{4}$

Lemma 2. Let $A S_{\vdash}=\langle\vdash, \overline{-}$, id $\rangle$ be an argumentation setting that is contrapositable and let $S$ be a set of $\mathcal{L}$-formulas. Then: $\operatorname{Grd}\left(A F\left(A S_{\vdash}(S)\right)\right) \subseteq \operatorname{Args}_{\vdash}(\operatorname{Free}(S)) .^{5}$

Proposition 2. Let $A S_{\vdash}=\langle\vdash, \bar{\cdot}, \mathrm{id}\rangle$ such that $(\vdash, \cdot)$ is contrapositable, let $S$ be a set of $\mathcal{L}$-formulas and suppose that $T \subseteq \mathcal{L}$ has to be skeptically enforced for Sem $\in$ $\{\mathrm{Grd}, \mathrm{Cmp}\}$. Then $\mathrm{T}$ cannot be enforced if there is no minimal strongly $A S_{\vdash}(S)$-consistent $S^{\prime} \subseteq \mathcal{L}$ such that $T \subseteq$ $\mathrm{CN}\left(S^{\star}\right)$, where $S^{\star} \subseteq S^{\prime} \cup S$ and $S^{\prime} \cup S^{\prime \prime}$ is strongly $A S_{\vdash}(S)-$ consistent for each $A S_{\vdash}$-consistent $S^{\prime \prime} \subseteq S$.

Proof. Let $\mathrm{AS}_{\vdash}=\langle\vdash, \bar{\cdot}$, id $\rangle$ such that $(\vdash, \bar{\cdot})$ is contrapositable, let $S \subseteq \mathcal{L}$ and suppose that $\mathrm{T} \subseteq \mathcal{L}$ has to be skeptically enforced. Moreover, suppose that for each minimal $S^{\prime} \subseteq \mathcal{L}$ such that there is some minimal $S^{\star} \subseteq S \cup S^{\prime}$ for which $\mathrm{T} \subseteq \mathrm{CN}\left(\mathrm{S}^{\star}\right)$, there is some $A S_{\vdash}$-consistent $\mathrm{S}^{\prime \prime} \subseteq \mathrm{S}$ such that $S^{\prime} \cup S^{\prime \prime}$ is $A S_{\vdash}$-inconsistent. It follows that for each $S^{\prime}$, there is some $\gamma \in S^{\prime}$ such that $\gamma \notin \operatorname{Free}\left(S \cup S^{\prime}\right)$. By assumption $S^{\prime}$ is minimal, from which it follows that $\gamma \in S^{\star}$ as well. Since $\operatorname{Grd}\left(A F\left(A S_{\vdash}\left(S \cup S^{\prime}\right)\right)\right) \subseteq \operatorname{Args}_{\vdash}(\operatorname{Free}(S \cup$ $\left.\left.S^{\prime}\right)\right)$, it follows that $\gamma \notin \operatorname{Supps}\left(\operatorname{Grd}\left(A \bar{F}\left(A S_{\vdash}\left(S \cup S^{\prime}\right)\right)\right)\right)$. Since $S^{\star}$ is minimal to obtain $\mathrm{T}$, it follows that $\mathrm{T} \nsubseteq$ $\operatorname{Concs}\left(\operatorname{Grd}\left(\operatorname{AF}\left(A_{\vdash}\left(S \cup S^{\prime}\right)\right)\right)\right)$.

The next example illustrates what happens when $\mathrm{T}$ is inconsistent with the current set of formulas $S$.

Example 8. Consider the setting $A S_{\mathrm{CL}}=\left\langle\vdash_{\mathrm{CL}}, \neg\right.$, id $\rangle$, let $S=\{p\}$ and suppose that $T=\{\neg p\}$ has to be enforced. Note that $S \cup \mathrm{T}$ is $A S_{\mathrm{CL}}$-inconsistent. We have that $\operatorname{Grd}\left(A F\left(A S_{\mathrm{CL}}(S \cup T)\right)\right)=\emptyset$ and that $\operatorname{Sem}\left(A F\left(A S_{\mathrm{CL}}(S \cup\right.\right.$ $T))=\left\{\left\{\operatorname{Args}_{\mathrm{CL}}(S)\right\},\left\{\operatorname{Args}_{\mathrm{CL}}(T)\right\}\right\}$ for Sem $\in\{$ Prf, Stb $\}$. Therefore, while $\mathrm{T}$ is credulously enforced, it is not skeptically enforced.

Consistent sets. Now we consider the relation between extensions and sets of formulas as shown in Proposition 1. In particular, any set of formulas $\mathrm{T}$ can be skeptically [resp. credulously] enforced if there is some set of $\mathcal{L}$-formulas $S^{\prime}$ such that $\mathrm{T}$ is part of the consequences of all [resp. some] maximal strongly $A S_{\vdash}(S)$-consistent subsets of $S \cup S^{\prime}$ :

Theorem 1. Let $A F\left(A S_{\vdash}(S)\right)$ be such that $A S_{\vdash}=\langle\vdash, \bar{\cdot}, \mathrm{id}\rangle$ is contrapositable and let $T \subseteq \mathcal{L}$ be the set that should be enforced. Then:

\footnotetext{
${ }^{4}$ In this section, where space allows we provide proof sketches of (parts of) the proofs. Full proofs can be found in the online technical appendix.

${ }^{5}$ Free $(S)$ is the set of $\mathcal{L}$-formulas from $S$ that is part of every $\mathrm{T} \in \operatorname{MCS}(\mathrm{S}): \operatorname{Free}(\mathrm{S})=\bigcap_{\mathrm{T} \in \operatorname{MCS}(\mathrm{S})} \mathrm{T}$.
} 
1. If $T$ can be skeptically enforced then there is some $S^{\prime} \subseteq \mathcal{L}$ such that $T \subseteq \mathrm{CN}\left(S^{\star}\right)$ and $S^{\star} \subseteq$ Free $\left(S \cup S^{\prime}\right)$ for $\operatorname{Sem} \in$ $\{\mathrm{Grd}, \mathrm{Cmp}\}$;

2. $T$ can be skeptically enforced iff there is some $S^{\prime} \subseteq \mathcal{L}$ such that for every $S^{\star} \in \operatorname{MCS}\left(S \cup S^{\prime}\right), T \subseteq \mathrm{CN}\left(S^{\star}\right)$ for Sem $\in\{$ Prf, Stb $\}$;

3. $T$ can be strictly skeptically enforced iff there is some $S^{\prime} \subseteq \mathcal{L}$ such that for every $S^{\star} \in \operatorname{MCS}\left(S \cup S^{\prime}\right), C N(T)=$ $\mathrm{CN}\left(S^{\star}\right)$ for Sem $\in\{$ Prf, Stb $\}$;

4. $T$ can be credulously enforced iff there is some $S^{\prime} \subseteq \mathcal{L}$ and some $S^{\star} \in \mathrm{CS}\left(S \cup S^{\prime}\right)$ such that $T \subseteq \mathrm{CN}\left(S^{\star}\right)$ for Sem $\in\{$ Cmp, Prf, Stb $\}$;

5. T can be strictly credulously enforced iff there is some $S^{\prime} \subseteq \mathcal{L}$ such that there is some $S^{\star} \in \operatorname{MCS}\left(S \cup S^{\prime}\right)$ such that $\mathrm{CN}(T)=\mathrm{CN}\left(S^{\star}\right)$ for Sem $\in\{$ Prf, Stb $\}$.

Proof. Let $\mathrm{AF}\left(\mathrm{A}_{\vdash}(\mathrm{S})\right)$ be such that $\mathrm{A} \mathrm{S}_{\vdash}=\langle\vdash, \overline{,}, \mathrm{id}\rangle$ is contrapositable and let $\mathrm{T} \subseteq \mathcal{L}$ be a set of formulas. We show Items 2 and 4:

2. $(\Rightarrow)$ Suppose that $\mathrm{T}$ can be skeptically enforced by expanding $S$ with $S^{\prime}$. Then, for each extension $\mathcal{E} \in \operatorname{Sem}\left(\operatorname{AF}\left(\operatorname{AS}_{\vdash}\left(S \cup S^{\prime}\right)\right)\right), \quad T \subseteq \operatorname{Concs}(\mathcal{E})$. By Proposition 1.2 it follows immediately that for any $\mathrm{S}^{\star} \in \operatorname{MCS}\left(\mathrm{S} \cup \mathrm{S}^{\prime}\right), \mathrm{T} \subseteq \mathrm{CN}\left(\mathrm{S}^{\star}\right)$, since $\operatorname{Args}_{\vdash}\left(S^{\star}\right) \in \operatorname{Sem}\left(\operatorname{AF}\left(\operatorname{AS}_{\vdash}\left(S \cup S^{\prime}\right)\right)\right)$ and $\operatorname{Concs}(\mathcal{E}) \subseteq$ $\mathrm{CN}\left(\operatorname{Args}_{\vdash}\left(\mathrm{S}^{\star}\right)\right)$.

$(\Leftarrow)$ Suppose that $\mathrm{T}$ cannot be skeptically enforced, then for each $S^{\prime} \subseteq \mathcal{L}$ there is some $\mathcal{E} \in \operatorname{Sem}\left(A F\left(A S_{\vdash}\left(S \cup S^{\prime}\right)\right)\right)$ such that for some $\phi \in \mathrm{T}, \phi \notin \operatorname{Concs}(\mathcal{E})$. By Proposition 1.4 $\operatorname{Supps}(\mathcal{E}) \in \operatorname{MCS}\left(S \cup S^{\prime}\right)$. Hence, for any $S^{\prime} \subseteq \mathcal{L}$, there is some $S^{\star} \in \operatorname{MCS}\left(S \cup S^{\prime}\right)$ such that for some $\phi \in \mathrm{T}, \phi \notin \mathrm{CN}\left(\mathrm{S}^{\star}\right)$.

4. $(\Rightarrow)$ Suppose that $\mathrm{T}$ can be credulously enforced. Then there is some $S^{\prime} \subseteq \mathcal{L}$, such that there is an extension $\mathcal{E} \in$ $\operatorname{Sem}\left(A F\left(A S_{\vdash}\left(S \cup S^{\prime}\right)\right)\right)$ for which $T \subseteq \operatorname{Concs}(\mathcal{E})$. Note that $\mathrm{T} \subseteq \mathrm{CN}(\operatorname{Supps}(\mathcal{E}))$. By Proposition 1.3 it follows that $\operatorname{Supps}(\mathcal{E})$ is consistent.

$(\Leftarrow)$ Suppose that there is some $S^{\prime} \subseteq \mathcal{L}$ and some $S^{\star} \in C S\left(S \cup S^{\prime}\right)$ such that $T \subseteq C N\left(S^{\star}\right)$. By Proposition 1.1 there is some $S^{\star} \subseteq S^{\#}$ such that $\operatorname{Args}_{\vdash}\left(S^{\#}\right) \in$ $\operatorname{Sem}\left(\operatorname{AF}\left(A S_{\vdash}\left(S \cup S^{\prime}\right)\right)\right)$. Since $T \subseteq C N\left(S^{\star}\right)$, for each $\phi \in \mathrm{T}$ there is some $\Gamma \subseteq \mathrm{S}^{\star} \subseteq \mathrm{S}^{\#}$ such that $\Gamma \vdash$ $\phi$. Therefore $(\Gamma, \phi) \in \operatorname{Args}_{\vdash}\left(\mathrm{S}^{\#}\right)$ and hence $\mathrm{T} \subseteq$ $\operatorname{Concs}\left(\operatorname{Args}_{\vdash}\left(S^{\#}\right)\right)$.

Example 9. (Example 7 continued) With the results from Theorem 1, the skeptical enforcement of $r$ in Example 7 is not surprising: $r \in \bigcap \operatorname{MCS}(\{p, q, \neg p \vee \neg q, r\})$. This also explains why $r$ cannot be strictly enforced: there is no maximally consistent subset of $S \cup\{r\}$ with $r$ as the only member.

Remark 2. In the proof for skeptical enforcement, the results from Items 2 and 4 of Proposition 1 were used, while for credulous enforcement, the results from Items 1 and 3 of Proposition 1 were used. Note that in Proposition 1, Items 1 and 3 are implied by Items 2 and 4 respectively. This means that the results for credulous enforcement are more generally applicable, but also that these results hold when only Items 2 and 4 of Proposition 1 are known for a setting.
Remark 3. In view of this theorem, one might suspect that enforcement in structured argumentation comes down to finding maximally consistent subsets of which the desired set is a consequence. However, some remarks are in order.

- As was shown in (Vesic and van der Torre 2012), it is possible to instantiate abstract argumentation with classical logic and obtain a meaningful setting (i.e., the rationality postulates from (Caminada and Amgoud 2007) are satisfied) of which the conclusions do not correspond to maximally consistent subsets. Therefore, argumentation is a richer formalism than reasoning with maximally consistent subsets and the result from Theorem 1 only illustrates the possibility of enforcement under these conditions. See (Arieli, Borg, and Heyninck 2019) for a more detailed discussion on and (Arieli, Borg, and Straßer 2021) for a characterization of the relation between structured argumentation and maximally consistent subsets.

- In this paper the results are about the (im)possibility of enforcement. When it comes to how a set of formulas should be enforced the implementation might yield a different result. For example, new information might be obtained over time in a dialogue setting (recall the online trade fraud example from Section 2). In such a case, the result of a question might be different from what was necessary to enforce a set of formulas and with that one answer the possibility of enforcement changes.

- Further possibilities to enforce sets of formulas are discussed in the remainder of the paper. For example, the results from Proposition 3 and Theorem 2 do not rely on the results from Proposition 1 nor is it assumed that the argumentation setup is contrapositable.

Acceptance relation. Next we consider monotonic acceptance relations: when new information is added the acceptance of the previously derived information does not change. While argumentation is mainly employed to model nonmonotonic reasoning, as Lemma 3 shows, not all acceptance relations are nonmonotonic. Proposition 3 is therefore relevant in our setting as well.

Definition 13 (Monotonic acceptance relation). Let $A F\left(A_{\vdash}(S)\right)$ be an argumentation framework. An acceptance relation is called monotonic iff for any set of formulas $T$, if $T$ is accepted in the framework, then $T$ is accepted in any expansion of that framework.

The next proposition shows that a (set of) formula(s) that can be derived from a consistent set of formulas can be enforced in an argumentation framework in which the acceptance relation is monotonic. We give an example of such a relation in Lemma 3.

Proposition 3. Let $A F\left(A S_{\vdash}(S)\right)$ be an argumentation framework for $A S_{\vdash}=\langle\vdash, \overline{,}, \mathrm{id}\rangle$ and suppose that the considered acceptance relation is monotonic. Then:

- Any $\phi \in \mathcal{L}$, such that $\Gamma \vdash \phi$ for an $A S_{\vdash}$-consistent $\Gamma \subseteq \mathcal{L}$, can be enforced by expanding $S$ with $\Gamma$; and

- Let $S^{\prime}$ be an $A S_{\vdash}$-consistent set of $\mathcal{L}$-formulas, such that $T \subseteq \mathrm{CN}\left(S^{\prime}\right)$. Then $T$ can be enforced by expanding the framework with $S^{\prime}$. 
Proof. Let $\mathrm{AF}\left(\mathrm{AS}_{\vdash}(\mathrm{S})\right)$ be an argumentation framework for $\mathrm{A}_{\vdash}=\langle\vdash, \overline{-}, \mathrm{id}\rangle$ with a monotonic acceptance relation. We show the first item, the proof of the second item is similar. Suppose that $\Gamma \vdash \phi$ for some strongly $\mathrm{A}_{\vdash}$-consistent $\Gamma \subseteq \mathcal{L}$ and that $\phi$ should be enforced. By Lemma 1 it follows that $\phi$ is accepted in $\operatorname{AF}\left(\mathrm{AS}_{\vdash}(\Gamma)\right)$. Since acceptance is assumed to be monotonic, $\phi$ is accepted in $\operatorname{AF}\left(A_{S_{\vdash}}(S \cup \Gamma)\right)$.

The above results hold for contrapositable frameworks and credulous acceptance:

Lemma 3. If $A F\left(A S_{\vdash}(S)\right)$ is an argumentation framework for a contrapositable setting $A S_{\vdash}=\langle\vdash,-$, id $\rangle$ then credulous acceptance is monotonic for Sem $\in\{\mathrm{Cmp}, \mathrm{Prf}, \mathrm{Stb}\}$.

Proof. Let $\mathrm{AF}\left(\mathrm{A}_{\vdash}(\mathrm{S})\right)$ be such that $\mathrm{AS}_{\vdash}=\langle\vdash, \overline{,}$, id $\rangle$ is contrapositable, let Sem $\in\{\mathrm{Cmp}$, Prf, Stb $\}$ and suppose that $\mathrm{T}$ is credulously accepted in $\operatorname{AF}\left(\mathrm{AS}_{\vdash}(\mathrm{S})\right)$. Moreover, let $S^{\prime} \subseteq \mathcal{L}$ be arbitrary. Since $\mathrm{T}$ is credulously accepted in $\operatorname{AF}\left(\bar{A} S_{\vdash}(S)\right)$, there is some $\mathcal{E} \in \operatorname{Sem}\left(A F\left(A_{\vdash}(S)\right)\right)$ such that $\mathrm{T} \subseteq \operatorname{Concs}(\mathcal{E})$. By Proposition $1.3 \operatorname{Supps}(\mathcal{E})$ is strongly $\mathrm{AS}_{\vdash}(\mathrm{S})$-consistent (i.e., $\operatorname{Supps}(\mathcal{E}) \in \mathrm{CS}(\mathrm{S})$ ) and thus, by Proposition 1.1 there is some $\operatorname{Supps}(\mathcal{E}) \subseteq \mathrm{T}^{\prime} \subseteq \mathrm{S} \cup \mathrm{S}^{\prime}$ such that $\operatorname{Args}_{\vdash}\left(T^{\prime}\right) \in \operatorname{Sem}\left(A F\left(A S_{\vdash}\left(S \cup \bar{S}^{\prime}\right)\right)\right)$. Note that $\mathrm{T} \subseteq \operatorname{Concs}\left(\operatorname{Args}_{\vdash}\left(\mathrm{T}^{\prime}\right)\right)$. Therefore $\mathrm{T}$ is credulously accepted in $\left.A F\left(A S_{\vdash}\left(S \cup S^{\prime}\right)\right)\right)$, for any $S^{\prime}$.

The next examples are counterexamples of acceptance relations that are not monotonic. The first shows that skeptical acceptance is non-monotonic.

Example 10. Consider the setting $A_{S_{\mathrm{CL}}}=\left\langle\vdash_{\mathrm{CL}}, \neg\right.$, id $\rangle$ and let $S=\{p\}$. Note that $p$ is a credulous consequence in $A F\left(A S_{\mathrm{CL}}(S)\right)$ and since credulous acceptance is monotonic (Lemma 3) $p$ will remain a credulous consequence. Let $\neg p$ be the formula that should be skeptically enforced. Note that, by Proposition 3, $\neg p$ is credulously enforced by expanding the framework with $S^{\prime}=\{\neg p\}$. However, then both $p$ and $\neg p$ will be credulously accepted in $A F\left(A S \mathrm{CL}\left(S \cup S^{\prime}\right)\right)$. Therefore, $\neg p$ will never be skeptically accepted.

The second example considers argumentation frameworks in which a preference relation is defined on arguments. Adding a preference relation is a common tool in the argumentation literature to refine conclusions, see e.g., (Amgoud and Cayrol 2002; Modgil and Prakken 2013). However, as the example will show, the credulous acceptance relation is no longer monotonic when preferences are added. In this paper we use the following notion of preferences among formulas and sets of formulas:

Definition 14 (Preference relation). Let $\mathcal{L}$ be a formal language, $v: \mathcal{L} \rightarrow \mathbb{N}$ a preference relation on formulas in $\mathcal{L}$ and let $\phi, \psi \in \mathcal{L}$. We denote that $\phi$ is [strictly] preferred over $\psi$ w.r.t. $v$ by $\left[\psi \prec_{v} \phi\right] \psi \preceq_{v} \phi$.

The preference relation can be lifted to sets of formulas, such that $S \subseteq \mathcal{L}$ is [strictly] preferred over $\mathrm{T} \subseteq \mathcal{L}$ w.r.t. $v$ is denoted by $\left[\mathrm{T} \prec_{v} \mathrm{~S}\right] \mathrm{T} \preceq_{v} \mathrm{~S}$. The formal definition of the lifting of the preference relation to sets of formulas is left abstract in this paper. Examples can be found in, e.g., (Modgil and Prakken 2013). An argument $a$ is preferred over an argument $b$ if $\operatorname{Supp}(a)$ is preferred over $\operatorname{Supp}(b)$, given some preference relation $v$. We accommodate preferences in the attack relation in the usual way (Amgoud and Cayrol 2002; Modgil and Prakken 2013): for $a, b, \in \operatorname{Args}_{\vdash}(\mathrm{S}), a \preceq-$ attacks $b$ iff $a$ attacks $b$ and $\operatorname{Supp}(b)$ is not strictly preferred over $\operatorname{Supp}(a)$.

Example 11. Let $A S_{\mathrm{CL}}=\left\langle\vdash_{\mathrm{CL}}, \neg\right.$, id $\rangle$ and $S=\{p\}$ as in the previous example and suppose that there is a preference relation $v$ defined such that $p \prec_{v} \neg p$. In the flat case, both $\neg p$ and $p$ are credulously accepted in the framework expanded with $\{\neg p\}$, since there was an argument $(\{\neg p\}, \neg p)$ which attacks and is attacked by $(\{p\}, p)$. However, in the prioritized case $(\{p\}, p)$ can no longer attack $(\{\neg p\}, \neg p)$ : there is no defense for the $\preceq$-attack from $(\{\neg p\}, \neg p)$. Hence $p$ is no longer accepted given this $v$.

Relevance. The results in (Borg and Straßer 2018), on non-interference (Caminada, Carnielli, and Dunne 2011), can also be used to say something about the possibility to enforce a (set of) formula(s). Intuitively, non-interference is a helpful property to have, since it indicates that adding irrelevant information does not change the acceptability of the already derived conclusions. In what follows let Atoms $(S)$ denote the set of atoms that occur in the formulas of $\mathrm{S}$. It is said that two sets of formulas $S_{1}$ and $S_{2}$ are syntactically disjoint (denoted by $\left.S_{1} \mid S_{2}\right)$ if Atoms $\left(S_{1}\right) \cap \operatorname{Atoms}\left(S_{2}\right)=\emptyset$.

Definition 15. (Non-interference, (Caminada, Carnielli, and Dunne 2011)) An acceptance relation satisfies noninterference iff for all $S_{1} \cup\{\phi\} \cup S_{2} \subseteq \mathcal{L}$ such that $\left(S_{1} \cup\{\phi\}\right) \mid S_{2}$ it holds that: $\phi$ is a consequence of $S_{1}$ iff $\phi$ is a consequence of $S_{1} \cup S_{2}$.

In (Borg and Straßer 2018) an extensive discussion can be found on the requirements on the elements of an argumentation setting in order for the acceptability relations to satisfy non-interference (e.g., the deducability relation is assumed to satisfy the basic relevance criterion from relevance logic (Avron 2014) and the target function is assumed to be monotonic (id is monotonic)). In a dynamic setting, we have the following result:

Theorem 2. Let $A F\left(A S_{\vdash}(S)\right)$ be such that $A S_{\vdash}=\langle\vdash, \bar{\cdot}, \mathrm{id}\rangle$ and that the acceptance relation satisfies non-interference. A set of formulas T can be credulously and skeptically enforced if there is some $A S_{\vdash}$-consistent $S^{\prime} \subseteq \mathcal{L}$ such that $T \subseteq \mathrm{CN}\left(S^{\prime}\right)$ and $S^{\prime} \mid S$.

Proof. Let $\mathrm{AF}\left(\mathrm{AS}_{\vdash}(\mathrm{S})\right)$ be an argumentation framework such that the considered acceptance relation satisfies noninterference. Moreover, let $S^{\prime}$ be a strongly $A_{S_{\vdash}}$-consistent set of $\mathcal{L}$-formulas, such that $S^{\prime} \mid S$ and let $T \subseteq$ $\mathrm{CN}\left(\mathrm{S}^{\prime}\right)$. Since $S^{\prime}$ is strongly $A S_{\vdash}$-consistent, it follows by Lemma 1 that $\mathrm{T}$ is credulously and skeptically accepted in $A F\left(A S_{\vdash}\left(S^{\prime}\right)\right)$. Since $S^{\prime} \mid S$ and the acceptance relation satisfies non-interference, it follows that $\mathrm{T}$ is credulously and skeptically accepted in $\mathrm{AF}\left(\mathrm{A} S_{\vdash}\left(S \cup S^{\prime}\right)\right)$ as well.

Expansion types. Although enforcement in structured argumentation is different from enforcement in abstract argumentation, the expansion types from (Baumann and Brewka 
2010) (recall Definition 7) are available in structured argumentation as well. Intuitively, the next proposition follows since the structure of the existing arguments does not change when an argumentation framework is expanded, hence there are no new attacks between the existing arguments after the expansion.

Proposition 4. Any expansion of an argumentation framework is normal.

Proof. Let $\mathrm{AF}\left(\mathrm{AS}_{\vdash}(\mathrm{S})\right)=\left\langle\operatorname{Args}_{\vdash}(\mathrm{S}), \mathrm{A}\right\rangle$ be an argumentation framework and suppose that $S$ is expanded by $S^{\prime}$. Let the result be $A F\left(A S_{\vdash}\left(S \cup S^{\prime}\right)\right)=\left\langle\operatorname{Args}{ }^{\star}, A^{\star}\right\rangle$, where $\operatorname{Args}^{\star}=\operatorname{Args}_{\vdash}\left(S \cup S^{\prime}\right)$ and $A^{\star}=\left\{(a, b) \in \operatorname{Args}^{\star} \times \operatorname{Args}^{\star} \mid\right.$ $a$ attacks $b\}$. Now suppose that $S^{\prime}$ is such that the expansion is not normal. Then there are $a, b \in \operatorname{Args}_{\vdash}(\mathrm{S})$ such that $(a, b) \in \mathrm{A}^{\star} \backslash \mathrm{A}$. However, since $a, b \in \operatorname{Args}_{\vdash}(\mathrm{S})$, by the definition of attack (Definition 3 ) it follows immediately that $a$ attacks $b$ in $A F\left(A S_{\vdash}(S)\right)$ as well. A contradiction.

The next two remarks discuss conditions on the argumentation setting and framework which ensure that an expansion is not only normal, but also weak or strong.

Remark 4. In some structured argumentation approaches (e.g., ABA (Bondarenko et al. 1997) and ASPIC ${ }^{+}$(Modgil and Prakken 2013)) it is possible to construct arguments that cannot be attacked since these are derived from strict rules and/or premises. On the one hand, if the framework before the expansion consists of such arguments, the expansion is weak: the existing arguments are not attacked. On the other hand, if the new arguments that can be created with the expansion cannot be attacked, the expansion is strong: only the existing arguments can be attacked.

Remark 5. Consider a language $\mathcal{L}$ and a preference relation $v$ for it. If an argumentation framework $A F\left(A S_{\vdash}(S)\right)$ is expanded in such a way that the new arguments are strictly stronger than the existing ones, the expansion is strong. If the framework is expanded such that the existing arguments are strictly stronger, the expansion is weak.

Monotonicity. In (Baumann and Brewka 2010) conditions on the semantics and the expansion type are studied to ensure that the expansion is monotonic, i.e., that the number of extensions does not decrease and the acceptance status of the already existing arguments does not change when a framework is expanded. In view of Proposition 1 and Theorem 1 we can show the structured counterpart of (Baumann and Brewka 2010, Theorem 5).

Proposition 5. Let $A F\left(A S_{\vdash}(S)\right)$ be an argumentation framework for a contrapositable $A S_{\vdash}=\langle\vdash,-, \mathrm{id}\rangle$ and let $S^{\prime}$ be an expansion. Then, for Sem $\in\{$ Prf, Stb $\}$ :

- $\left|\operatorname{Sem}\left(A F\left(A S_{\vdash}(S)\right)\right)\right| \leq\left|\operatorname{Sem}\left(A F\left(A S_{\vdash}\left(S \cup S^{\prime}\right)\right)\right)\right|$;

- $\forall \mathcal{E} \in \operatorname{Sem}\left(A F\left(A S_{\vdash}(S)\right)\right), \exists \mathcal{E}^{\prime} \in \operatorname{Sem}\left(A F\left(A S_{\vdash}\left(S \cup S^{\prime}\right)\right)\right)$ such that $\mathcal{E} \subseteq \mathcal{E}^{\prime}$.

Proof sketch. Let $\mathrm{AF}\left(\mathrm{AS}_{\vdash}(\mathrm{S})\right)$ be an argumentation framework for a contrapositable $A S_{\vdash}$ and let $S^{\prime}$ be an expansion. Note that MCS is monotonic: for every $\mathrm{T} \in \operatorname{MCS}(\mathrm{S})$, there is some $\mathrm{T} \subseteq \mathrm{T}^{\prime} \in \operatorname{MCS}\left(\mathrm{S} \cup \mathrm{S}^{\prime}\right)$.
- This follows since $\left|\operatorname{Stb}\left(A F\left(A_{\vdash}(\mathrm{T})\right)\right)\right| \leq$ $\left|\operatorname{Prf}\left(\mathrm{AF}\left(\mathrm{A}_{\vdash}(\mathrm{T})\right)\right)\right|$ for any set of formulas $\mathrm{T}$ (i.e., every stable extension is a preferred extension (Dung 1995)). Since, $|\operatorname{MCS}(S)| \leq\left|\operatorname{MCS}\left(S \cup S^{\prime}\right)\right|$, $\left|\operatorname{Prf}\left(\operatorname{AF}\left(\mathrm{AS}_{\vdash}(\mathrm{S})\right)\right)\right| \leq|\mathrm{MCS}(\mathrm{S})|$ (Proposition 1.4) and $\left|\operatorname{MCS}\left(S \cup S^{\prime}\right)\right| \leq\left|\operatorname{Stb}\left(A F\left(A S_{\vdash}\left(S \cup S^{\prime}\right)\right)\right)\right|$ (Proposition 1.2).

- Let $\mathcal{E} \in \operatorname{Sem}\left(A F\left(A_{\vdash}(S)\right)\right)$. Then, by Proposition 1.4 it follows that $\operatorname{Supps}(\mathcal{E}) \in \operatorname{MCS}(S)$. Hence, there is some $\mathrm{T} \in \operatorname{MCS}\left(\mathrm{S} \cup \mathrm{S}^{\prime}\right)$ such that $\operatorname{Supps}(\mathcal{E}) \subseteq \mathrm{T}$. By Proposition 1.2 it follows that $\operatorname{Args}_{\vdash}(\mathrm{T}) \in \operatorname{Sem}\left(\mathrm{AF}\left(\mathrm{A} S_{\vdash}(\mathrm{S} \cup\right.\right.$ $\left.\left.S^{\prime}\right)\right)$ ) (recall that every stable extension is also preferred). Since $\operatorname{Supps}(\mathcal{E}) \subseteq \mathrm{T}, \mathcal{E} \subseteq \operatorname{Args}_{\vdash}(\mathrm{T})$. Because $\mathcal{E}$ was arbitrary, this holds for all Prf-and Stb-extensions.

Remark 6. (Baumann and Brewka 2010, Theorem 5) contains a third item (which serves as the converse of the second item) that in a structured setting does not hold without further restrictions. Consider again Example 10, we have that $\operatorname{Prf}\left(A F\left(A S_{\mathrm{CL}}(\{p\})\right)\right)=\left\{\operatorname{Args}_{\vdash}(\{p\})\right\}$, while $\operatorname{Prf}\left(A F\left(A S_{\mathrm{CL}}(\{p, \neg p\})\right)\right)=\left\{\operatorname{Args}_{\vdash}(\{p\}), \operatorname{Args}_{\vdash}(\{\neg p\})\right\}$. Note that the extension Args $s_{\vdash}(\{\neg p\})$ does not extend an extension in the framework before the expansion.

\section{Discussion}

Throughout the paper some assumptions were made to show the results. We will discuss some of these in this section, to illustrate that such restrictions are common in the literature.

In many of the results it was assumed that the target function is id. When looking at well-known approaches to structured argumentation (Besnard et al. 2014) and the translation of these approaches as provided by (Borg and Straßer 2018) it can be seen that the target function is often id. In ABA and $\mathrm{ASPIC}^{+}$this is always the case, because of the translation, and in logic-based argumentation attack rules such as direct defeat and direct undercut are covered by id.

The first formal results required that the argumentation setting was contrapositive. In, e.g., (Heyninck and Arieli 2018; Modgil and Prakken 2013) the same assumption is made to show, e.g., the rationality postulates from (Caminada and Amgoud 2007) and the relation to reasoning with maximally consistent subsets. As we consider it essential that these rationality postulates are satisfied, the argumentation setting would be contrapositive in most settings anyway.

Like in (Borg and Straßer 2018), we do not consider a preference ordering on the arguments. However, throughout the paper some remarks were already made about preference orderings; we plan to formally investigate the use of preferences in future work.

\section{Related Work}

Enforcement is an important research direction in the study of dynamics in formal argumentation (Doutre and Mailly 2018). In (Baumann and Brewka 2010) (im)possibility results are shown for the enforcement of extensions, based on abstract principles of semantics, rather than specific Dungstyle semantics. Moreover, conditions under which the addition of new arguments and attacks between them does 
not change the acceptance status of already existing arguments are investigated as well. Other, related questions have been investigated such as extension removal (Baumann and Brewka 2019), enforcement by changing the semantics (Doutre and Mailly 2017) and the minimal change necessary to enforce an extension (Baumann 2012; CosteMarquis et al. 2014; Wallner, Niskanen, and Järvisalo 2017), all for abstract argumentation frameworks (Dung 1995).

An enforcement operator for structured argumentation is introduced in (Wallner 2020). However, the question of enforcement is still at the argument level: how to adjust the knowledge base such that a certain (set of) argument(s) becomes a part of some extension. In our paper we are interested in enforcement on the formula level. This might lead to different conclusion, since, for example, there might be more than one argument for a certain formula.

In a structured argumentation setting we are not aware of any further research on enforcement. There is, however, some work on other aspects of dynamics. In (Alfano et al. 2018), an incremental approach to efficiently recalculating the acceptability status of literals in updated DeLP programs is introduced, in (Modgil and Prakken 2012) resolutions of attacks are studied in the context of $\mathrm{ASPIC}^{+}$, and in (Testerink, Odekerken, and Bex 2019; Odekerken, Borg, and Bex 2020) the stability of the acceptability status of a certain formula is investigated. Finally, (Booth et al. 2014) study concepts related to enforcement in (abstract) abductive argumentation frameworks and show that these frameworks can be instantiated by abductive logic programming.

By definition, enforcement is related to belief revision (Alchourrón, Gärdenfors, and Makinson 1985; Katsuno and Mendelzon 1991). This relation has been surveyed in, e.g., (Falappa, Kern-Isberner, and Simari 2009; Falappa et al. 2011) where it is argued that formal argumentation and belief revision model two complementary aspects of human commonsense reasoning. In particular, where belief revision concerns how an agent's beliefs can be updated consistently, argumentation is concerned with the process of inferring beliefs, and the reasons for and against these beliefs (Falappa, Kern-Isberner, and Simari 2009, p. 355).

In the dynamic argumentation literature, belief revision (like) postulates have been employed to handle changes in formal argumentation. We mention some general trends. Many dynamic argumentation approaches that are combined with belief revision postulates are based on a translation of the argumentation framework and notions such as acceptance and Dung-style semantics into logical formulas. For example, (de Saint-Cyr et al. 2016) introduce a new logical language called YALLA, (Doutre, Herzig, and Perrussel 2014; Doutre, Maffre, and McBurney 2017) use the Dynamic Logic of Propositional Assignments and (Baumann and Brewka 2015) introduce so-called Dung-logics (see (Doutre and Mailly 2018) for a more detailed overview). Belief revision has also been combined with existing structured argumentation approaches. For example, (Snaith and Reed 2016) adjust the belief revision postulates to account for the structure of $\mathrm{ASPIC}^{+}$elements, thus modelling dynamic $\mathrm{ASPIC}^{+}$. Furthermore, non-prioritized belief revision is modelled in (Shakarian et al. 2016) for a probabilistic variation of DeLP, and revision in logic-based argumentation is modeled in (Krümpelmann et al. 2012). Each of these examples studies the question how to revise the argumentation framework to obtain good conclusions, which is the central question in belief revision. In contrast, enforcement as studied in this paper is useful for structured argumentation applications based on dialogues, which are exactly the kind of applications that have been implemented at the Dutch National Police and in other application domains, see e.g., (Chalaguine et al. 2018; Visser, Lawrence, and Reed 2020).

\section{Conclusion and Future Work}

In this paper we have formulated enforcement for a structured setting: given a set of formulas $\mathrm{T}$, is it possible to expand the knowledge base such that $\mathrm{T}$ becomes credulously or skeptically accepted? We discussed a real-life application where this question is relevant (Section 2) and argued why enforcement in structured argumentation should be about formulas (Example 5). For the general approach to structured argumentation from (Borg and Straßer 2018), we have studied a variety of conditions under which a set of formulas $\mathrm{T}$ can be (strictly) credulously/skeptically enforced: (maximal) consistency of the expansion, relevance, and monotonicity of the acceptance relation. Moreover, we have shown that, although the setting and the notion of enforcement is different from (Baumann and Brewka 2010), any expansion is normal and satisfies some monotonicity properties. While some of our results build on existing literature, we have shown that the existing studies on static argumentation settings can be employed in dynamic settings as well. This suggests that the extensive research on static structured argumentation can be generalized to dynamic setting, which makes the research better applicable.

As far as we know, this is the first study on enforcement of sets of formulas in a general structured argumentation setting. This opens up the possibility to further investigate dynamics for specific structured settings (Besnard et al. 2014; Bondarenko et al. 1997; Modgil and Prakken 2013), and to extend the investigation of other notions of dynamics from knowledge representation in formal argumentation.

In addition to extending the current setting with preferences, in future work we will also look into other approaches to structured argumentation that are not covered by the general setting from (Borg and Straßer 2018) (e.g., DeLP (García and Simari 2004) and claim-augmented argumentation frameworks (Dvořák and Woltran 2020)). Moreover, we will study how a set of formulas can be enforced. To this end, we will study different aspects of enforcement for structured argumentation, such as the minimal change necessary to enforce a set of formulas, efficient enforcement, removal of formulas for enforcement, and enforcing the removal of sets of formulas from the conclusion. While some of these more computational aspects of enforcement can be studied in the general setting, for others (e.g., efficient algorithms or complexity studies), we will have to look into specific approaches to structured argumentation. 


\section{Acknowledgements}

This research has been partly funded by the Dutch Ministry of Justice and the Dutch National Police.

\section{References}

Alchourrón, C.; Gärdenfors, P.; and Makinson, D. 1985. On the logic of theory change: Partial meet contraction and revision functions. Journal of Symbolic Logic 50(2):510530.

Alfano, G.; Greco, S.; Parisi, F.; Simari, G. I.; and Simari, G. R. 2018. An incremental approach to structured argumentation over dynamic knowledge bases. In Proceedings of KR'18, 78-87.

Amgoud, L., and Cayrol, C. 2002. A reasoning model based on the production of acceptable arguments. Annals of Mathematics and Artificial Intelligence 34(1-3):197-215.

Arieli, O.; Borg, A.; and Heyninck, J. 2019. A review of the relations between logical argumentation and reasoning with maximal consistency. Annals of Mathematics and Artificial Intelligence 87(3):187-226.

Arieli, O.; Borg, A.; and Straßer, C. 2021. Characterizations and classifications of argumentative entailments. In Proceedings of the 18th International Conference on Principles of Knowledge Representation and Reasoning (KR'21). To appear.

Avron, A. 2014. What is relevance logic? Annals of Pure and Applied Logic 165(1):26-48.

Baumann, R., and Brewka, G. 2010. Expanding argumentation frameworks: Enforcing and monotonicity results. In Proceedings of COMMA'10, 75-86. IOS Press.

Baumann, R., and Brewka, G. 2015. AGM meets abstract argumentation: Expansion and revision for Dung frameworks. In Yang, Q., and Wooldridge, M. J., eds., Proceedings of the 24th International Conference on Artificial Intelligence (IJCAI'15), 2734-2740. AAAI Press.

Baumann, R., and Brewka, G. 2019. Extension removal in abstract argumentation - an axiomatic approach. In Proceedings of AAAI'19, 2670-2677. AAAI press.

Baumann, R. 2012. What does it take to enforce an argument? Minimal change in abstract argumentation. In Proceedings of ECAI'12, 127-132. IOS Press.

Besnard, P.; García, A.; Hunter, A.; Modgil, S.; Prakken, H.; Simari, G.; and Toni, F. 2014. Introduction to structured argumentation. Argument \& Computation 5(1):1-4.

Bex, F.; Testerink, B.; and Peters, J. 2016. AI for online criminal complaints: From natural dialogues to structured scenarios. In Workshop proceedings of Artificial Intelligence for Justice at ECAI 2016, 22-29.

Bondarenko, A.; Dung, P. M.; Kowalski, R.; and Toni, F. 1997. An abstract, argumentation-theoretic approach to default reasoning. Artificial Intelligence 93(1):63-101.

Booth, R.; Gabbay, D.; Kaci, S.; Rienstra, T.; and van der Torre, L. 2014. Abduction and dialogical proof in argumentation and logic programming. In Schaub, T.; Friedrich, G.; and O'Sullivan, B., eds., Proceedings of ECAI'14, 117-122. IOS Press.
Borg, A., and Straßer, C. 2018. Relevance in structured argumentation. In Proceedings of $I J$ CAI'18, 1753-1759. ijcai.org. Extended version at: http://arxiv.org/abs/1809.04861.

Brewka, G.; Thimm, M.; and Ulbricht, M. 2019. Strong inconsistency. Artificial Intelligence 267:78-117.

Caminada, M., and Amgoud, L. 2007. On the evaluation of argumentation formalisms. Artificial Intelligence 171(5):286 - 310 .

Caminada, M.; Carnielli, W.; and Dunne, P. 2011. Semi-stable semantics. Journal of Logic and Computation 22(5):1207-1254.

Chalaguine, L. A.; Hadoux, E.; Hamilton, F.; Hayward, A.; Hunter, A.; Polberg, S.; and Potts, H. W. W. 2018. Domain modelling in computational persuasion for behaviour change in healthcare. CoRR abs/1802.10054.

Coste-Marquis, S.; Konieczny, S.; Mailly, J.-G.; and Marquis, P. 2014. On the revision of argumentation systems: Minimal change of arguments statuses. In Proceedings of KR'14, 52-61. AAAI Press.

Čyras, K.; Oliveira, T.; Karamlou, A.; and Toni, F. 2020. Assumption-based argumentation with preferences and goals for patient-centric reasoning with interacting clinical guidelines. Argument \& Computation.

de Saint-Cyr, F. D.; Bisquert, P.; Cayrol, C.; and LagasquieSchiex, M.-C. 2016. Argumentation update in YALLA (yet another logic language for argumentation). International Journal of Approximate Reasoning 75:57-92.

Doutre, S., and Mailly, J.-G. 2017. Semantic change and extension enforcement in abstract argumentation. In Proceedings of SUM'17, 194-207. Springer.

Doutre, S., and Mailly, J.-G. 2018. Constraints and changes: A survey of abstract argumentation dynamics. Argument \& Computation 9(3):223-248.

Doutre, S.; Herzig, A.; and Perrussel, L. 2014. A dynamic logic framework for abstract argumentation. In Baral, C.; De Giacomo, G.; and Eiter, T., eds., Proceedings of the 14th International Conference on Principles of Knowledge Representation and Reasoning (KR'14), 62-71. AAAI Press.

Doutre, S.; Maffre, F.; and McBurney, P. 2017. A dynamic logic framework for abstract argumentation: Adding and removing arguments. In Benferhat, S.; Tabia, K.; and Ali, M., eds., Proceedings of the 30th International Conference on Industrial, Engineering, Other Applications of Applied Intelligent Systems (IEA/AIE'17), volume 10351 of Lecture Notes in Computer Science, 295-305. Springer.

Dung, P. M. 1995. On the acceptability of arguments and its fundamental role in nonmonotonic reasoning, logic programming and $\mathrm{n}$-person games. Artificial Intelligence 77(2):321-357.

Dvořák, W., and Woltran, S. 2020. Complexity of abstract argumentation under a claim-centric view. Artificial Intelligence 285:103290.

Falappa, M.; García, A.; Kern-Isberner, G.; and Simari, G. 2011. On the evolving relation between belief revi- 
sion and argumentation. The Knowledge Engineering Review 26(1):35-43.

Falappa, M. A.; Kern-Isberner, G.; and Simari, G. R. 2009. Belief Revision and Argumentation Theory. Springer. 341360.

García, A., and Simari, G. 2004. Defeasible logic programming: an argumentative approach. Theory and Practice of Logic Programming 4(1\&2):95-138.

Heyninck, J., and Arieli, O. 2018. On the semantics of simple contrapositive assumption-based argumentation frameworks. In Modgil, S.; Budzynska, K.; and Lawrence, J., eds., Proceedings of the 7th International Conference on Computation Models of Argument (COMMA'18), Frontiers in Artificial Intelligence and Applications 305, 9-20. IOS Press.

Katsuno, H., and Mendelzon, A. 1991. On the difference between updating a knowledge base and revising it. In Allen, J. F.; Fikes, R.; and Sandewall, E., eds., Proceedings of the 2nd International Conference on Principles of Knowledge Representation and Reasoning (KR'91), 387-394. Morgan Kaufmann.

Krümpelmann, P.; Thimm, M.; Falappa, M.; García, A.; Kern-Isberner, G.; and Simari, G. R. 2012. Selective revision by deductive argumentation. In Proceedings of TAFA'11, 147-162. Springer.

Modgil, S., and Prakken, H. 2012. Resolutions in structured argumentation. In Proceedings of COMMA'12, 310-321. IOS Press.

Modgil, S., and Prakken, H. 2013. A general account of argumentation with preferences. Artificial Intelligence 195:361-397.

Odekerken, D., and Bex, F. 2020. Towards transparent human-in-the-loop classification of fraudulent web shops. In Villata, S.; Harašta, J.; and Křemen, P., eds., Proceedings of the 33rd Conference on Legal Knowledge and Information Systems (JURIX'20), volume 334 of Frontiers in Artificial Intelligence and Applications, 239-242. IOS Press.

Odekerken, D.; Borg, A.; and Bex, F. 2020. Estimating stability for efficient argument-based inquiry. In Prakken, H.; Bistarelli, S.; Santini, F.; and Taticchi, C., eds., Proceedings of the 8th International Conference on Computational Models of Argument (COMMA'20), volume 326 of Frontiers in Artificial Intelligence and Applications, 307-318. IOS Press.

Prakken, H. 2010. An abstract framework for argumentation with structured arguments. Argument \& Computation 1(2):93-124.

Prakken, H. 2020. An Argumentation-Based Analysis of the Simonshaven Case. Topics in Cognitive Science - Special Issue on Rational Models of Legal Proof 12(4):1068-1091.

Shakarian, P.; Simari, G.; Moores, G.; Paulo, D.; Parsons, S.; Falappa, M. A.; and Aleali, A. 2016. Belief revision in structured probabilistic argumentation. Annals of Mathematics and Artificial Intelligence 78(3):259-301.

Snaith, M., and Reed, C. 2016. Argument Revision. Journal of Logic and Computation 27(7):2089-2134.
Testerink, B.; Odekerken, D.; and Bex, F. 2019. A method for efficient argument-based inquiry. In Proceedings of FQAS'19, 114-125. Springer.

Vesic, S., and van der Torre, L. W. N. 2012. Beyond maxiconsistent argumentation operators. In del Cerro, L. F.; Herzig, A.; and Mengin, J., eds., Proceedings of the 13th Conference on Logics in Artificial Intelligence, (JELIA'12), volume 7519 of Lecture Notes in Computer Science, 424436. Springer.

Visser, J.; Lawrence, J.; and Reed, C. 2020. Reasonchecking fake news. Communications of the ACM 63(11):38-40.

Wallner, J.; Niskanen, A.; and Järvisalo, M. 2017. Complexity results and algorithms for extension enforcement in abstract argumentation. Journal of Artificial Intelligence Research 60:1-40.

Wallner, J. 2020. Structural constraints for dynamic operators in abstract argumentation. Argument \& Computation 11(1-2):151-190.

Zeng, Z.; Shen, Z.; Tan, B. T. H.; Chin, J. J.; Leung, C.; Wang, Y.; Chi, Y.; and Miao, C. 2020. Explainable and Argumentation-based Decision Making with Qualitative Preferences for Diagnostics and Prognostics of Alzheimer's Disease. In Proceedings of the 17th International Conference on Principles of Knowledge Representation and Reasoning, 816-826. 